\title{
Enfoques teóricos sobre la participación educativa en la vejez
}

\author{
ANTONIO VÍCTOR MARTÍN \\ Universidad de Salamanca
}

\begin{abstract}
SUMMARY.-The purpose of this article is to examine and to estract of differents approaches and theoretical models about aging process, the more important outcomes for educative practice with regard to the educative participation of the older adults.

The perspectives of differents approach such as biologic and behavioral perspective; Lifespan model; Disengagement theory; Activity theory; Subcultural theory; and Continuity theory are studied. Finally, the new gerontologies approaches concerning with relativization of the influence on educative participation factors age-asociated are discussed.
\end{abstract}

\section{INTRODUCCIÓN}

La importancia ponderal de la educación sobre el envejecimiento exitoso de los individuos crea, entre otras, la necesidad de enfatizar los factores relacionados con la participación de éstos en actividades socioeducativas, especialmente aquellos factores y causas que distinguen a los individuos ancianos participantes de los no participantes. A lo largo de los últimos veinticinco años, la literatura científica gerontológica se ha ocupado y preocupado en desarrollar un cuerpo de conocimientos sobre los factores estimuladores o inhibidores asociados a dicha participación. Principalmente, ésta se ha analizado en términos de considerar la influencia de determinados factores motivacionales (Boshier (1978) ${ }^{1}$; Burguess (1972)2; Morstain y Smart (1974)3 ; Peterson (1981) ${ }^{4}$; Pearce

1. Boshier, R. (1971). Motivational Orientation of Adult education participants: A factor Analytic exploration of Houle's Tipology. Adult Education Journal, V. XXI, $N^{\circ} 2, p p .3-26$.

2. Burguess (1972). Reasons for adult participation in group educational activities. Adult Education, 22, pp. 3-9.

3. Mostain, B. R. y Smart, J. C. (1974). Reasons for participation in adult education courses: A multivariate analysis of group differences. Adult Education, 24, pp. 83-98.

4. Peterson, D. A. (1981). Participation in education by older people. Educational Gerontology, 7, pp. 245-256. 
(1991) $)^{5}$; etc.); determinados factores actitudinales y/o sociodemográficos asociados al seguimiento de cursos, programas, etc. (Beder, 1990) ${ }^{6}$; Dellmann-Jenkins; Fruit y Lambert $(1984)^{7}$; Heisel $(1980 ; 1981)^{8}$; etc.; en términos de barreras o impedimentos a la participación educativa (Darkenwald y Valentine $(1985)^{9}$; Hayes $(1988)^{10}$; Scanland, y Darkenwald (1984) ${ }^{11}$; Valentine y Darkenwald (1990) ${ }^{12}$; Martindale y Drake (1989) ${ }^{13}$; etc.); o, incluso, en términos de analizar la propia naturaleza de los mismos procesos de envejecimiento, como veremos más adelante.

A pesar de este esfuerzo, la dispersidad y, en muchos casos, disparidad de los resultados empíricos obtenidos hacen necesario también el considerar los presupuestos teóri$\cos$ de partida donde se han agrupado y agrupan muchos de estos resultados. El propósito de las líneas que siguen por tanto es el de resaltar, de manera sistematizada, las líneas teóricas que han presidido gran parte de estos planteamientos y que han condicionado, en no pocas ocasiones, las diversas visiones sobre la vejez en general, y sobre la participación educativa de ancianos en particular.

\section{La PARTICIPACIÓN SOCIOEdUCATIVA DE ANCIANOS Y PERSPECTIVA DERIVADA DE LA ORIENTACIÓN BIOLÓGICO-CONDUCTUAL}

La participación de ancianos en cualquier tipo de actividad y, entre ellas, las actividades educativas, ha estado mediatizada, en la investigación gerontológica tradicional, por la concepción, en cierto modo determinística, de los, por otro lado evidentes, deterioros físicos y biológicos que acompañan al paso de los años. De este modo, la edad cronológica han sido relacionada con la generalización universal de distintas disminuciones y pérdidas físicas (problemas respiratorios; cardiovasculares, déficits sensoriomotores, pérdida de agudeza visual o auditiva...) y psíquicas en la vejez (déficits en los procesos cognitivos como la memoria, la atención,...) lo que conduce, frecuentemente, a

5. Pearce, D. S. (1991). Toward understanding the participation of older adults in continuing education. Educational Gerontology, 17, pp. 451-464.

6. Beder, H. (1990). Reasons for nomparticipation in Adult Basic Education. Adult Education Quarterly, V. 40, $n^{\circ} 4$, pp. 207-218.

7. Dellmann-Jenkins; Fruit, D. y Lambert, D. (1984). Exploring age integration in the University classroom: middle age and younger student's educational motives and instructional preferences. Educational Gerontology, v. 10, $n^{\circ}$ 6, pp. 429-440.

8. Heisel, A. M. (1980). Adult participation and the disadvantaged older adult: an analytical review of the research literature. Educational Gerontology, V. 5. $n^{\circ} 2$, pp. 125-137.

9. Darkenwald, G. G.; Valentine, T. (1985). Factor structure of deterrents to public participation in adult education. Adult Education Quarterly, 35, pp. 177-193.

10. Hayes, R. E. (1988). A typology of low-literate adults based on perceptions of deterrents to participation in adult basic education. Adult Education Quarterly, V. 39, $n^{\circ} 39, p p .1-10$.

11. Scanlan, C. L. y Darkenwald, G. G. (1984). Identifying deterrents to participation in continuing education. Adult Education Quarterly, V. 34, pp. 155-166.

12. Valentine y Darkenwald (1990). Deterrents to participation in adult education: profiles of potencial learners. Adult Education Quarterly, V. 41, $n^{\circ} 1, p p .29-42$.

13. Martindale, J. C.; Drake, B. J. (1989). Factor structure of deterrents to participation in off-duty adult education programs. Adult Education Quarterly, 39, $n^{\circ} 2$, pp. 63-75. 
considerar dichos déficits como potenciales inhibidores de conductas de participación efectiva en programas socioeducativos.

Esta perspectiva 'biologicista', presente durante mucho tiempo en numerosos trabajos de investigación, está siendo, poco a poco, modificada, sobre todo a partir de los años setenta, por los resultados de investigaciones donde se ha puesto en evidencia gran parte de los métodos de medición y diagnóstico empleados en la valoración de las respuestas de los sujetos. Métodos en los que la velocidad de ejecución o el tiempo de reacción han marcado negativamente los resultados de los sujetos ancianos frente a sujetos más jóvenes; donde la identificación y/o categorización de las informaciones recibidas no eran suficientemente significativas ni familiares a los viejos, y donde, consiguientemente con lo anterior, la rapidez en la toma de decisiones de éstos estaba muy por debajo de los parámetros normales para individuos más jóvenes.

El avance de la investigación gerontológica, en algunos casos por medio de estudios comparativos realizados en diferentes grupos de población, en su mayoría con demostrados y conocidos déficits en su funcionamiento intelectivo, han introducido nuevas variables explicativas y moduladoras que acompañan a la variable edad y a los deterioros físico-biológicos que parecen estar más ampliamente relacionados con otras variables como por ejemplo los estados carenciales de salud, los efectos de la institucionalización en centros residenciales o asistenciales, o la disminución de interacciones sociales.

En definitiva, parece aceptado hoy día que existen innegables limitaciones en los niveles de ejecución cognitivos y motores, así como deterioros físicos progresivos en los organismos, que son inherentes a los procesos de envejecimiento pero que, y al contrario de lo que se creía hasta hace unos años, estas limitaciones no invalidan irremediable y universalmente los niveles de actividad física y/o mental de los individuos por muy avanzada que sea su edad. Incluso existen algunas investigaciones que muestran la reversibilidad del declive universal relacionado con la edad biológica en aspectos como la capacidad cerebral, el tiempo de reacción (Baltes, Hoyer y Labouvie, 1973 ${ }^{14}$; Murell, 197015; etc.) o, la reversibilidad de los deterioros en cuestiones como la capacidad de trabajo físico o los déficits sensoriales.

La cuestión tal vez esté en facilitar a las personas ancianas adecuados ambientes donde éstas puedan trabajar a ritmos individuales de funcionamiento físico y/o mental ${ }^{16}$.

\section{LA PARTICIPACIÓN SOCIOEDUCATIVA DE ANCIANOS Y LA PERSPECTIVA DERIVADA DE LAS TEORÍAS SOCIOAMBIENTALES}

En gran parte influidas por el auge de la Gerontología Social sobre todo en los años 70 , las teorías socioambientales del envejecimiento han enfatizado el impacto de las

14. Baltes, P. B. y Labouvie, G. V. (1973). Adult development of intellectual performance: description, explanation and modification. En Eisdorfer, C. y Lawton, M. P. (Eds.) (1973): The Psychology of adult development and aging. Washington: American Psychological Association.

15. Murrell, F. H. (1970). The effect of extensive practice on age differences in reaction time. Journal of Gerontology, 25, pp. 268-274.

16. Véase: Baltes, M. M. y Barton, M. E. (1977). New Approaches toward aging: a case for the operant model. Educational Gerontology, $n^{o}$ 2, pp. 383-405. 
condiciones ambientales sobre los individuos ancianos. Autores como Gubrim (1972) ${ }^{17}$; Dowd (1975) ${ }^{18}$; Baltes, 1973; Baltes y Schaie, 1973; 1976; Goulet y Baltes, 1970; Nesslroade y Reese, 1974...) son muestra de esta perspectiva. En este caso, también la edad cronológica ha jugado un peso específico importante dentro de las definiciones sociológicas sobre el envejecimiento al determinar gran parte de los eventos funcionales y conductuales que se dan en la vida de una persona en interacción con los otros (Neugarten, Moore y Lowe, 1965) ${ }^{19}$. Así, institucional, legal y socialmente el comienzo de la vejez se establece en los 65 años de edad, que, generalmente y hasta hoy, marca el momento final del período laboral activo y da paso a la jubilación. A partir de ese momento y sin que haya ninguna otra justificación que la de 'tener la edad', se producen diversas circunstancias, vividas con desigual intensidad por cada uno de los sujetos jubilados, pero que, en términos generales, suponen la pérdida, por parte de éste, del papel de trabajador como miembro activo del sistema ocupacional, lo que se había convertido poco menos que en la esencia de la identidad personal del individuo hasta entonces. Este hecho también conlleva a súbitas rupturas con las costumbres básicas de la vida hasta entonces desarrollada; la modificación de los ritmos diarios y de ocupación del tiempo libre y del ocio, generalmente dependiente de las situaciones laborales, y ahora, falto de estructura y organización; así como a la disminución de la frecuencia de los contactos y la solidaridad con el grupo de colegas y compañeros de trabajo. Por otro lado, la modificación de otros roles principales como el de padre (o madre) coincidiendo con el final de la etapa de crianza y dependencia de los hijos hacia los padres se torna, en muchos casos, en dependencia de éstos hacia aquellos (pasando a un segundo plano de importancia el rol de padre, sustituyéndose en algunos casos por el de abuelo y en ocasiones acompañado de la crianza directa de los nietos); $y$, por último, la disolución del vínculo matrimonial por la muerte de uno de los cónyuges, lo que supone un verdadero mazazo en la estructura vital del superviviente.

Todo este conjunto de acontecimientos vitales a los que algunos autores han dado en llamar «eventos o sucesos vitales» o «carreras normativas», definidos como las transiciones de carácter positivo o negativo que se producen en la etapa adulta y que producen ciertos cambios en las pautas vitales del individuo, junto con otras variables socioambientales como el estatus socioeconómico, los contextos étnicos, religiosos y socioculturales, han sido estudiados como potenciales inhibidores de la participación social de las personas (participación política, cultural, educativa ...)

De este modo, tanto el ambiente social como el propio ambiente físico han sido considerados como funcionalmente importantes en el proceso de iniciación y en el mantenimiento de determinadas conductas de participación educativa. En el primer caso, tanto las actitudes, las creencias y/o imágenes mentales hacia la misma actividad educativa, la percepción de los ancianos como estudiantes o participantes en educación, o hacia las propias posibilidades y capacidades de éstos, mantenidas tanto por el grupo de ancianos

17. Gubrium, J. F. (1972). Toward a socio-environmental theory of aging. The Gerontologist, 12, pp. 281-284.

18. Dowd, J. J. (1975). Aging as exhange: A preface to theory. Journal of Gerontology, 30, pp. 584-594.

19. Neugarten, B.; Moore, J. W. y Lowe, J. C. (1965). Age norms, age constraints and adult socialization. American Journal of Sociology, 70, pp. 710-717. 
como del resto de la comunidad, son factores determinantes. Así mismo, y en el segundo caso, el ambiente físico ha recibido también un pormenorizado interés en cuanto a su influencia como inhibidor o potenciador de la participación social y educativa de los suje$\operatorname{tos}^{20}$. Algunos autores como Rebok $(1981)^{21}$ consideran que el conocimiento de la participación educativa adulta se desarrolla de acuerdo con factores dependientes de factores ambientales, físicos y situacionales, más que únicamente en factores relacionados con un inexorable y biológico declive. El ambiente físico proporciona, en numerosas ocasiones y como indicábamos, más barreras que facilidades a la participación. Entre éstas, las más frecuentemente mencionadas por los propios ancianos, son las dificultades de transporte; mal acondicionamiento de locales, falta de luminosidad, problemas de temperatura, etc. (March, Hooper y Baum, 1977)22.

Por otro lado, y como señalábamos más atrás, las teorías socioambientales del envejecimiento han remarcado la importancia sociológica no sólo de la edad cronológica (al destacar ésta los eventos funcionales y conductuales en la vida de una persona, Neugarten, Moore y Lowe, $1965^{23}$ ), sino también de factores tan relevantes como la viudez y la jubilación, considerados como factores que afectan a la participación social de los ancianos (Fischgrund, 1976 ${ }^{24}$ ).

La importancia de los planteamientos socioambientales en la explicación del fenómeno del envejecimiento es manifestada por Baltes y Barton (1977) indicando que este tipo de teorías han contribuido a que:

a) la vejez haya sido incluida dentro de las teorías del comportamiento.

b) los procesos del envejecimiento sean percibidos como un complejo fenómeno en el que tiene cabida tanto los determinantes genético-biológicos, como los ambientales. En este sentido, argumentan que los cambios desde un modelo basado en la enfermedad, en la dolencia o en el mal, de un modelo que describe el envejecimiento básicamente como un declive intrínseco, biológicamente fijado, a un modelo ambiental/comportamental, que enfatiza la interacción entre el organismo y sus ambientes externos e internos, necesita de un nuevo marco explicativo de las relaciones funcionales para los eventos conductuales y los ambientes.

20. Cabe destacar aquí el enfoque del estudio de los condicionantes contextuales desde una perspectiva ecológico-conductual, representado en España por la profesora Rocío Fernández Ballesteros, con algunos estudios en ancianos sobre la influencia directa de determinadas características físicas y de percepción de ambientes en la potenciación o inhibición de determinadas características conductuales. Valgan como ejemplo: Fernández Ballesteros, R.; Díaz Veiga, P.; Izal y Fernández de Troconiz, M.; y González, J. L. (1987): Evaluación de una residencia de ancianos y valoración de intervenciones ambientales. En Fernández Ballesteros, R. (1987): El Ambiente. Análisis Psicológico. Ed. Pirámide. Madrid.

21. Rebok, W. G. (1981). Aging and higher education: Prospects for intervention. Educational Gerontology, 6, pp. 39-48.

22. March, G. B.; Hooper, J. O. y Baum, J. (1977). Life span education and the older adult: Living is learning. Educational Gerontology, 2, pp. 163-172.

23. Neugarten, B.; Moore, J. W. y Lowe, J. C. (1965). Op. cit., p. 715.

24. Fischgrund, E. (1976). Social participation and social integration of the aged: Implications for social welfare. Journal of Social and Social Welfare, 3, 4, Mar. pp. 409-420. 
Para estos autores, tal red teórica debe requerir:

a) la descripción del ecosistema existente donde se desarrolla, y funciona la persona anciana.

b) el diseño de óptimos ecosistemas para mantener, rehabilitar y promover un funcionamiento y nivel óptimo de realización del individuo anciano (Baltes y Barton, $\left.1977^{25}\right)$.

\section{LA PARTICIPACIÓN SOCIOEDUCATIVA DE ANCIANOS Y LA PERSPECTIVA DERIVADA DE LOS PLANTEAMIENTOS CONDUCTUAL-OPERANTE}

Es de sobra conocido que gran parte del énfasis prestado a la necesidad de análisis funcionales sobre la interdependencia establecida entre los elementos del binomio individuo-ambiente (o medio) se ha debido a la aparición y desarrollo del conductismo y, más en concreto, del conductismo instrumental u operante con Skinner a la cabeza. Baltes y Barton (1977) destacan dos principios de aprendizaje que, a su vez se convierten en dos premisas básicas del modelo operante y que tienen aplicación para el estudio de la conducta de participación educativa:

1. Plasticidad conductual: es decir, desde el conocido planteamiento conductual, la mayoría de las conductas son aprendidas, incluyendo las de participación activa en actividades educativas, la de salud, la de enfermedad e incluso la conducta de «ser y hacer de viejo», y son susceptibles de corrección, modificación, optimización y prevención en todos los grupos de edad. Esto va en contra del principio de que el declive es universal e irreversible.

2. Interdependencia dinámica: esto es, los eventos conductuales pueden sólo ser descritos y explicados en conjunción con variables ambientales. Este principio está en consonancia con el argumento anterior en el sentido que se destaca que la capacidad de ejecutar ciertas tareas o destrezas tiene su origen en la interacción entre variables tales como la propia habilidad, la motivación, etc. y las condiciones concretas de la situación donde se realicen ${ }^{26}$.

De este modo, y dado que una conducta instrumental $u$ operante se halla regida por sus consecuencias ambientales, la adquisición, mantenimiento, extinción y/o modificación de la conducta de participación está, desde este punto de vista, sujeta a una combinación de las siguientes contingencias conductuales:

a) La presencia de consecuencias ambientales aversivas que pueden tener lugar si se realiza dicha conducta (aquí intervendrían numerosos mitos y prejuicios sociales existentes sobre la tercera edad, falsas expectativas, la presencia de determinadas dolencias físicas interpretadas como un resultado de la realización de la conducta, etc.).

b) La pérdida o ausencia de estímulos discriminativos que den lugar a que la conducta ocurra. En este último caso, y dentro de la misma órbita lingüística y conceptual, la

25. Baltes, M. M.; Barton, M. E. (1977). Op. cit., pp. 383-405.

26. Baltes, M. M.; Barton, M. E. (1977). Op. cit., p. 387. 
pérdida o ausencia de contingencias positivas puede ser atribuida a algunos cambios de tipo intrínseco o a cambios de tipo extrínseco que acontecen en la vejez. Por ejemplo, la pérdida de refuerzos positivos hacia la conducta de participación puede ser el reflejo de una pérdida o ausencia de agentes reforzadores (cónyuge, familiares, amigos, etc.) y de ambientes desfavorables (regímenes de institucionalización, residencias, asilos, etc.). Así como debidos a cambios intrínsecos como variaciones en las necesidades y/o los intereses educativos de los ancianos, etc.

Por consiguiente, las condiciones ambientales, ecológicas, consideradas incluso desde un punto de vista puntualmente lineal como estímulos, tanto de carácter positivas como negativas, parecen tener amplia credibilidad como factores influyentes en la emisión de conductas de participación en actividades socioeducativas.

$\mathrm{El}$ reconocimiento de la perspectiva operante como un puntal importante en el avance de la investigación gerontológica puede concretarse en dos aportes importantes:

a) El examen del grado de reversibilidad de las conductas en la vejez y las condiciones por las que la reversibilidad puede ser llevada a cabo; esto es, la consideración del tipo de antecedentes que son necesarios para disponer las condiciones necesarias para que la capacidad de ejecutar ciertas conductas ocurra y el examen del tipo de refuerzos necesarios para poder aumentar y/o mantener esta ejecución en los viejos.

b) Desde un punto de vista metodológico, la utilización de la investigación observacional que permite una descripción sistemática de las relaciones funcionales existentes entre las condiciones ambientales y las conductas que son mostradas por los ancianos (Baltes y Barton, 1977: 388).

A caballo entre las teorías socioambientales y el enfoque conductual operante, se encuentran otra serie de perspectivas y modelos interactivos acogidos a fundamentaciones teóricas más amplias de las que también pueden deducirse consecuencias útiles y valiosas y que están desarrollando interesantes planteamientos y líneas de investigación dentro del ámbito gerontológico. Así, disciplinas como por ejemplo: la Psicología ambiental (con el estudio del individuo en relación a su medio construido); la Psicología Ecológica (con el estudio de los llamados 'escenarios de conducta'); el enfoque Cognitivo-Perceptivo (estudios sobre reacciones perceptivas al ambiente; mapas cognitivos sobre determinados espacios físicos como barrios, ciudades, residencias de ancianos, etc.); la Ecología Social (fundamentalmente nos referimos a los trabajos de R. Moos, 1973; Moos 1974; Moos e Insel, 1974, basados en el estudio del «clima social» en determinados contextos y/o ambientes ${ }^{27}$ ) o la reformulación de ésta; esto es, el llamado por Fernández Ballesteros 'Conductismo Ecológico', centrado en el análisis de las relaciones funcionales entre el ambiente y la conducta (F. Ballesteros, $1987^{28}$ ), etc.

De tal modo son importantes gran parte de las aportaciones derivadas de las concepciones anteriores, que la mayor parte de los autores estiman que cualquier estudio o cualquier intervención socioeducativa realizados sobre un mesosistema o exosistema (en

27. Véase, por ejemplo: Moos, H. R. (1973). Conceptualizations of human environments. American Psychologist, 28, pp. 652-665; Moos, H. R. (1974). Evaluating treatment environments: A social ecology approach. Wiley, New York; Moos, H. R. y Lemke, S. (1979): multiphasic environmental assesment procedure: preliminary manual. Social Ecology Laboratory, Stanford University. Palo Alto; etc.

28. Fernández Ballesteros, R. (1987). Op. cit., p. 29. 
el sentido dado por Brofenbrenner ${ }^{29}$ ) como el constituido por las residencias de ancianos, precisa de análisis descriptivos referenciales al contexto situacional donde se desarrollan las interacciones entre los individuos entre sí y de éstos con sus espacios percibidos, sociales y físicos ${ }^{30}$.

\section{LA PARTICIPACIÓN SOCIOEDUCATIVA DE ANCIANOS Y LA PERSPECTIVA DERIVADA DEL MODELO DE «LIFESPAN»}

La aparición de una serie de volúmenes en los años 70 bajo el título original de «Life Span Development Psychology» (Baltes, 1973; Baltes y Schaie 1973; 1976; Goulet y Baltes, 1970; Nesslroade y Reese, 1974; Decrow, 1974; Eisdorfer y Lawton, 1973; Peterson, 1975; Shaie, 1974; Wass y West, 1977; Taunb, 1980; etc.) ${ }^{31}$ marcaba la aceleración de un movimiento iniciado muchos años antes y fundamentado en numerosos trabajos desarrollados en norteamérica por autores tan importantes como Robert Havighurst; Erik Erikson (que con su teoría de los ocho estadíos ampliaba (entre otras cosas) significativamente el punto de mira de los estadios evolutivos freudianos a la edad adulta); o los estudios de la investigadora Bernice Neugarten hacia el diseño del ciclo vital humano como proceso desarrollado a lo largo de toda la vida; proceso vital en el que la importancia de las etapas primeras del desarrollo no es considerada absoluta y determinante ni fruto de una sucesión ontogenética, sino relativa y sumativa a posteriores etapas. La edad cronológica como unidad de análisis evolutivo prioritario dejaba paso a la consideración de la importancia de factores biográficos individuales, es decir, la historia de eventos o sucesos notables importantes en la vida de las personas, conjugando éstos con eventos normativos históricos que afectan a determinadas generaciones o cohortes de población.

De este modo, se justificaba el intento de estudiar los procesos madurativos básicos a través de todo el ciclo vital con una clara configuración interdependiente entre lo social y lo psicológico (Maddox y Wiley, 1976) ${ }^{32}$.

29. Brofembrenner, U. (1987). La Ecología del desarrollo humano. Ed. Paidós. Barcelona.

30. Pueden servir como ilustración a esto algunos trabajos dirigidos por la profesora Fernández Ballesteros como por ejemplo: Fernández Ballesteros, R. y González, J. L.; Izal, M. V. y Espinosa, M. J. (1982). Influencia del mobiliario en la conducta interpersonal de ancianos institucionalizados. En Fernández Ballesteros, R. (Ed.) (1982). Evaluación de Contextos. Servicio de Publicaciones Universidad de Murcia. Murcia. En el mismo lugar: Fernández Ballesteros, R.; Izal, M. V.; Díaz, P.; González Sánchez, J. L.; Vila Abad, E.; y Juan Espinosa, M. (1982). Estudio ecopsicológico de una residencia de ancianos, etc.

31. De modo tan sólo testimonial algunos de los autores más representativos de esta serie de volúmenes sobre el Lifespan son: Buhler, C. y Massarik, F. (Eds.) (1968). The course of human life. N. York Springer; Palmore, E. y Jeffers, F. C. (Eds.) (1971). Prediction of Life Span. Massachussets. Heath.; Datan, N. y Grinsberg, L. (Eds.) (1975). Life-Span developmental psychology. Normative Life crisis; y sobre todo: Baltes, P. B. (Ed.) (1978). Life Span development and behavior. Vol. 1. Academic Press. N. York. ...; Baltes, P. B. y Brim, O. G. (Eds.) (1983). Life Span development psychology, Vol. 5, Academic Press. N. York.

32. Maddox, L. G. y Wiley, J. (1976). Scope, Concepts and Methods in the study of aging. Cap. I, p. 24. En Binstock, H. R. y Shanas, E. (Eds.) (1976). Handboock of Aging and the Social Sciences. Litton educational Publishing. Inc. VRN. New York. 
El énfasis sobre la enorme relevancia prestada a lo generacional y a los efectos cohorte junto a eventos no normativos, individuales, frente a las diferencias de edad, en cuanto a las inevitables comparaciones entre individuos jóvenes y viejos en lo que se refiere al grado de ejecución o realización de determinadas destrezas y/o habilidades, ha supuesto, a la par que nuevos enfoques en los métodos de investigación en y sobre los procesos de envejecimiento, nuevas conceptualizaciones sobre los determinantes que influyen en las conductas que realizan las personas ancianas.

Los nuevos enfoques derivados de esta perspectiva han propiciado también el apoyo a la tesis que mantiene la importancia de los antecedentes motivacionales y educativos frente a las relaciones entre los cambios biológico/maduracionales y los cambios comportamentales (Baltes y Barton, 1977) ${ }^{33}$.

De este modo se ha encontrado evidencia del impacto de variables no cognitivas, motivacionales y situacionales tales como por ejemplo la fatiga (Furrey y Baltes, 1973) ${ }^{34}$; la ansiedad ante la tarea; el enfrentarse a situaciones educativas novedosas (Boulet, 1972); etc., que pueden explicar las diferencias detectadas en muestras de sujetos con edades diferentes en el logro de ciertas ejecuciones y en la capacidad para responder con éxito a algunas pruebas y tests.

A pesar de la importancia de los avances que suponen los nuevos planteamientos introducidos por los teóricos de la psicología del desarrollo del ciclo vital, aparecen aquí algunas cuestiones insuficientemente explicadas y matizadas. De este modo, las críticas efectuadas a estos planteamientos van dirigidas fundamentalmente a la idea de que éstos no responden totalmente a planteamientos comprehensivos; es decir, no explican suficientemente algunas cuestiones como por ejemplo el hecho de que dentro de cada etapa vital, la conducta y los roles definidos como apropiados son bastante diversos y vividos con desigual intensidad por cada individuo; del mismo modo, para una misma edad, las variaciones ante los mismos estímulos son bastante diferentes.

Desde el terreno educativo, la perspectiva del ciclo vital tiende a considerar frecuentemente a la educación de una manera instrumental-adaptativa. De este modo, el argumento más claro que se exhibe es que los ancianos que participan en actividades educativas estarán mejor adaptados a sus ambientes, mientras que aquellos que no participen social y educativamente, tendrán mayores problemas para ajustarse a su medio. Con estar de acuerdo con la idea, entendemos, sin embargo, que existen muchas otras razones para participar en y de la educación que las meramente adaptativas.

Por otro lado, los teóricos del 'lifespan' tienden a dirigir la educación hacia el estado o etapa particular de la vida en que se encuentra el individuo, bien es verdad que destacando las posibilidades y potencialidades de las personas en cada momento de su ciclo vital. Este hecho, sin embargo puede tener un efecto negativo al servir para limitar la participación de las personas ancianas en programas educativos dirigidos a otros sectores de edad diferente.

33. Baltes, M. M.; Barton, M. E. (1977). Op. cit., pp. 385-387.

34. Furry, C. A. y Baltes, P. B. (1973). The effect of age differences in ability-extraneous performance variables on the assessment of intelligence in children, adults, and the elderly. Journal of Gerontology, 28, pp. 73-80. 
5. La Participación SOCIOEdUCATIVA de ANCIANOS Y LA PERSPECTIVA DERIVADA DE LA TEORÍA DE LA DESVINCULACIÓN

Esta teoría propuesta por Cumming y Henry en $1961^{35}$, percibe la vejez como un tiempo durante el cual el individuo gradual y voluntariamente se desvincula o va abandonando el interés y el desempeño de algunos roles y estatus. La reducción de la actividad es bien recibida por los ancianos y considerada como normal.

Dos nociones son de especial relevancia para esta teoría. Primera, la noción de que el aumento de interacción declina con la edad (Cumming y Henry, 1961: 46). Y segunda, el concepto de sincronicidad.

En el primer caso, no sólo las relaciones interpersonales se reducen en cantidad sino que aquellas que permanecen están alteradas en calidad. La disminución de los contactos sociales es bien visto por los ancianos porque supone una cierta liberación en cuanto a funciones y roles y a compromisos sociales que pueden suponer incomodidad. De este modo, los procesos de desvinculación son entendidos con el significado de que el anciano cede el testigo, transmite el poder de su generación a la siguiente, lo que incidentalmente redunda en un beneficio para toda la sociedad.

El segundo se refiere a la sincronización en el tiempo entre los cambios sociales y los personales. Si el anciano se «desvincula» de sus roles antes de tiempo, antes de estar preparado, se crea un desequilibrio. La persona está entonces 'asincronizada'. Lo mismo sucede si el viejo desempeña roles prescritos para jóvenes.

De este modo, la desvinculación de roles acompasados y el desempeño de roles aparecen como tópicos centrales de la teoría de la desvinculación.

Posiblemente podríamos decir que esta teoría ha recibido tanto o más críticas (Maddox, 1964 ${ }^{36}$; Atchely, 197237; McMordie, 1981 ${ }^{38}$; Covey, 1983; etc.) que apoyos. En primer lugar, algunos autores entienden que es incompleta en su covertura (Dowd, 1975: 585) $)^{39}$ y que la desvinculación temporal es selectiva; es decir, el individuo puede desvincularse en algunas actividades y vincularse, «engancharse» en otras. Las respuestas individuales al envejecimiento son muy variadas (Covey, 1983) ${ }^{40}$. En definitiva, se trata de lo que Havinghurst (1964) denominó: «desvinculación-vinculación-selectiva».

En segundo lugar, se argumenta que la vinculación más que la desvinculación está más fuertemente relacionada con el éxito del envejecimiento y el bienestar psicológico (Lipman y Smith, 1968: 521) ${ }^{41}$; Tobin y Neugarten $(1961)^{42}$.

35. Cumming, E. y Henry, W. E. (1961). Growing Old: The process of Disengagement. Basic Books. New York. (reeditado en: ARVO, New York, 1979). 82.

36. Maddox, G. L. (1964). Disengagement theory: A critical evaluation. Gerontologist, 4, pp. 80-

37. Atchley, R. C. (1972). Disengagement among professors. Journal of Gerontology, 26, 476-480.

38. McMordie, R. W. (1981). The Phenomenon of withdrawal in relation to the aging process: A development perspective. Social Behavior and Personality; v. 9. $n^{\circ} 1, p p .71-79$.

39. Dowd, J. J. (1975). Op. cit., pp. 584-594.

40. Véase Covey, H. C. (1983). Higher Education and Older People. Some theoretical considerations, Part. II. Educational Gerontology, 9, pp. 95-109.

41. Limpman, A. y Smith, K. J. (1968). Functionality of disengagement in Old Age. Journal of Gerontology, 23, pp. 517-523.

42. Tobin, S. S. y Neugarten, B. L. (1961). Life Satisfaction and social interaction in the aging. Journal of Gerontology, 16, pp. 344-346. 
Finalmente, se mantiene que la teoría es aplicable solamente a una parte de la población, que la noción de la irreversibilidad del declive es cuestionable y la asunción de la degeneración lineal discutible.

En suma, las críticas de la teoría de la Desvinculación cuestionan si la desvinculación es un inevitable, universal y progresivo proceso lineal y si la edad es un factor crucial o es un factor relativo incidental que abarca un complejo conjunto de variables (Covey, 1983: 96).

Aunque ya no es ampliamente aceptada esta teoría, si ha tenido un gran impacto sobre las teorías sociales del envejecimiento y sobre la práctica educativa.

Desde este último punto de vista, si interpretáramos estrictamente este tipo de presupuestos, tendríamos que considerar que:

$1^{\circ}$. El pretender que los ancianos adquieran o mantengan el rol de estudiantes, de participantes en educación, iría en contra de sus propios intereses y deseos íntimos, puesto que lo que realmente desean éstos precisamente es abandonar, desvincularse de los roles desempeñados y buscar la paz y la tranquilidad. La participación, de este modo, se entiende que, si se produce, responde más a consideraciones de «deseabilidad social» que a la propia voluntad y deseo personal del anciano. Esto es, el deseo de utilidad que pueden manifestar determinados individuos ancianos responde más a un intento de mitigar el temor de verse rechazados que al valor en sí mismo del propio deseo de sentirse útiles.

$2^{\circ}$. Por otro lado, se entiende también desde esta perspectiva que, funcionalmente, la participación en educación de los jubilados, no sirve a las necesidades de la sociedad, puesto que ellos no contribuyen ya a satisfacer éstas; y por tanto, los recursos humanos y materiales deben dirigirse hacia las poblaciones jóvenes.

$3^{\circ}$. Una tercera consecuencia que puede extraerse de aquí, en la que coinciden la mayor parte de los críticos a la teoría, es que el fenómeno de abandono, de desvinculación que exhiben los ancianos es simplemente un reflejo de la reducción de las oportunidades ambientales para la interacción social. De este modo, la intervención gerontoeducativa debería tender a reequilibrar, compensar y aumentar la calidad y cantidad de ese tipo de oportunidades procedentes del medio.

\section{LA PARTICIPACIÓN SOCIOEDUCATIVA DE ANCIANOS Y LA PERSPECTIVA DERIVADA DE LA TEORÍA DE LA ACTIVIDAD}

Diametralmente opuesta a la anterior, la teoría de la actividad fue propuesta en primer lugar por los autores americanos Havighurst y Albrecht, y sobre todo por Tartler $(1961)^{43}$ y más recientemente por Lemon, Bengston y Peterson (1972) ${ }^{44}$ y Knapp $(1977)^{45}$. En general, todos los gerontólogos educativos aceptan, de algún modo, este

43. Tartler, R. (1961). Das Alter in der modernen Gessellschaft. Enke, Sttutgart.

44. Lemon, B. W., Bengtson, V. L. y Peterson, J. A. (1972). An exploration of the activity theory of aging: Activity types and life expectation among inmovers to a retirement community. Journal of Gerontology, 27, pp. 511-523.

45. Knapp, M. R. J. (1977). The activity theory of aging: an examination in the English context. Gerontologist, 17, pp. 553-559. 
planteamiento, en el sentido de destacar la importancia y la necesidad del mantenimiento de actividad en cualquier etapa de la vida.

En palabras de Ursula Lehr $(1981)^{46}$ esta teoría parte de la hipótesis de que sólo es feliz y se siente satisfecha la persona que es activa, que produce algún rendimiento y que es útil a otros. Por contra, aquellas que no son o no se sienten «útiles», no desempeñan roles ni funciones relevantes, se sentirán poco ajustadas, desadaptadas e insatisfechas.

La teoría de la actividad parte de la idea de que los ancianos deben mantener su acostumbrado nivel de vinculación y de actividad y que el grado de vinculación o desvinculación con la sociedad está muy influenciado por los antecedentes experienciales de la persona. En definitiva se argumenta que el mantenimiento de sustanciales niveles de actividad son necesarios para un envejecimiento exitoso.

Desde un punto de vista educativo, supone la puesta en marcha de programas de activación, de participación en actividades sociales y educativas con el fomento de dinámicas grupales que «reactiven»e incentiven al anciano a realizar cosas. Desde esta perspectiva se considera que el entrenamiento físico, la activación de diversas energías corporales y el fomento del rendimiento somático está demostrado como un excelente medio de geroprofilaxis y tratamiento terapéutico ${ }^{47}$.

Se argumenta, en definitiva, que la salud, incluso en su faceta puramente fisiológica, debe aumentar con la realización de determinadas actividades físicas y/o mentales que aparecen como imprescindibles para el desarrollo de la persona.

A pesar de la evidencia de la bonanza de la actividad física y mental, la teoría de la actividad ha recibido también algunas críticas que se dirigen hacia la idea, presente en esta teoría, de que lo que el anciano realmente quiere es emular actividades, funciones y papeles de personas de menor edad, puesto que al realizar muchas de estas actividades, el anciano puede sentirse «más joven»; es decir, se critica el excesivo énfasis en la deseabilidad de papeles y funciones socialmente consideradas como propias de personas más jóvenes, como una especie de intento de retornar a épocas más activas, que suponían una mayor interacción social y una mayor autorrealización personal. El envejecimiento es visto así, como una lucha, una batalla por continuar en la edad madura, la edad inmediatamente anterior a la vejez. Es decir, la crítica fundamental a la teoría de la actividad es el considerar que ésta lo que realmente intenta es eliminar los patrones de conducta y roles peculiares de la vejez (Atchely, 1972: 205) (8) $^{48}$ sustituirlo por otros más propios de épocas anteriores. Por consiguiente se asume que los roles desempeñados en la madurez, son mucho más deseables que los roles desempeñados en la propia vejez.

Desde el polo opuesto entonces, el hecho de que algunos ancianos quisieran disminuir su nivel de actividad, relacionada con el desempeño de ciertos roles que anteriormente desempeñaban en una edad previa, llega a ser visto, desde este planteamiento, como problemático y no normativo.

Los detractores de la teoría entienden, además, que la participación educativa, (claramente fomentada por los partidos de la actividad) es vista como un intento de volver atrás en el tiempo, del mantenimiento de roles antiguos e impropios ya de la edad que se

46. Lehr, U. (1981). Op. cit., p. 245.

47. Algunos estudios empíricos como Jokl, 1954; Steinbach, $(1970,1971)$ han encontrado evidencia en este sentido. Véase Lehr, U. (1981). Op. cit., pp. 246-248.

48. Atchley, R. C. (1972). The Social forces in later life. Belmont. California. 
tiene, al tiempo que se entiende que la participación en educación supone, más que, buscar el valor de ésta en sí misma, una forma o un medio de sustituir, reemplazar otro tipo de actividades que fueron más provechosas pero que, con la jubilación, se han perdido (actividades laborales por ejemplo).

Por otro lado, otras críticas se dirigen a la consideración de que, en muchas ocasiones, la planificación de actividades en base a la activación de la persona se realiza sin criterios de racionalidad e intencionalidad pedagógicos claros. Se trata de que el anciano «haga cosas» por sí mismas, sin más.

En definitiva, se critica la no existencia de evidencia empírica de que el ideal del anciano sea el desempeñar papeles similares a los anteriormente tenidos; el concebir a la persona anciana como insatisfecha con su edad, el no valorar los aspectos deseables de la vejez y que parece negar que la vejez sea una época de crecimiento potencial en sí misma para la persona.

\section{La Participación SOCIOEdUCATIVA DE ANCIANOS Y LA PERSPECTIVA DERIVADA DE LA TeORÍA DE LA SUBCUltura}

Propuesta en primer lugar por Rose y Peterson $(1965)^{49}$. Se argumenta aquí que los ancianos, como grupo, tienen muchas de las características propias que definen la constitución de una subcultura. De este modo, se defiende que los ancianos, por lo general, están excluidos de modo implícito de la interacción y la participación en la sociedad; comparten características que trascienden a la sociedad y tienen una conciencia de grupo e intereses comunes que les mantienen unidos. En suma, los ancianos se identifican con otros ancianos como grupo, y además se ven forzados a actuar e interactuar entre ellos (Atchley, 1972: 159). Puesto que no puede hablarse precisamente de grupo privilegiado, el ser viejo puede conducir a la adquisición de una cierta autoconciencia de pertenencia a un grupo marginal y marginado, lo que enlazaría con la Teoría de la/s Minoria/s ( $\ll i$ nority Theory») estrechamente relacionada con la Teoría de la Subcultura, y que sostiene que los viejos comparten características con otros grupos minoritarios que son objeto de prejuicios y discriminación (por ejemplo: negros, gitanos, homosexuales, indios, etc.).

Las críticas efectuadas a la teoría de la subcultura van en la línea de la consideración de las escasas evidencias empíricas existentes sobre el hecho de que muchos ancianos se identifiquen a sí mismos como viejos (Blau, 197350; Palmore y Maddox, 197751) y del hecho de que los ancianos compartan intereses comunes o se adapten a problemas comunes a su grupo de iguales.

Desde el punto de vista educativo, si se acepta esta teoría las instituciones educativas tenderían a ofertar programas educativos específicamente diseñados para ancianos (de hecho, en nuestra opinión, este es precisamente uno de los «males» en los que ha caído la educación en la vejez).

49. Rose, A. M. y Peterson, W. A. (1965). Older People and their social world: The subculture of the aging. Philadelphia: F. A. Davis.

50. Blau, Z. S. (1973). Old age in a changing society. New York: Franklin Watts.

51. Palmore, E. B. y Maddox, G. L. (1977). Sociological aspects of aging. En W. Busse y E. Pfeiffer (Eds.) (1977). Behavior and adaptation in later life. Boston little, Brown. 
Para Covey (1983) si consideramos, según este planteamiento, la creencia de que los ancianos mantienen intereses, necesidades y valores comunes, entonces la participación de los ancianos no sería posible puesto que hoy día la asunción, identificación e interiorización del rol y función de 'estudiante', está asociado a otro grupo de edad diferente, y por tanto, el viejo al no pertenecer a ese grupo, tendría que asumir e identificarse con otro tipo de roles más apropiados a la vejez.

De este modo, se entiende que la teoría de la subcultura puede ser tachada de incompleta puesto que no da una respuesta suficiente del porqué real de la participación educativa de los viejos.

\section{La PARTICIPACIÓN SOCIOEdUCATIVA DE ANCIANOS Y LA PERSPECTIVA DERIVADA DE LA TEORIA DE LA CONTINUIDAD}

Ha sido una de las teorías más prometedoras en el campo de la Gerontología. Atchely (1971: 13) ${ }^{52}$ la define así: «La Teoría de la Continuidad defiende que en el proceso que conduce a ser adulto, el individuo desarrolla hábitos, cometidos, preferencias y una multitud de otras disposiciones que llegan a formar parte de su personalidad. A medida que el individuo envejece, se está predisponiendo para mantener contínuos estos hábitos, asociaciones y preferencias».

A diferencia de la Teoría de la Actividad, esta teoría no asume que los roles y las necesidades sean reemplazadas, sino que mantiene que la reacción de los individuos al envejecimiento puede ser explicado por el examen de complejas interrelaciones entre los cambios biológicos y psicológicos; los hábitos de las personas, sus preferencias y sus asociaciones; las oportunidades situacionales para la continuidad de estos hábitos; y su experiencia presente.

Las experiencias vitales de la persona a lo largo de su desarrollo crean, de este modo, ciertas predisposiciones, recursos, contactos, hábitos, etc., que se mantendrán en lo posible en el tiempo y que el individuo utilizará a lo largo de toda su vida.

Los cambios producidos en los sujetos son, de este modo, procesos adaptativos que abarcan las interacciones entre todos estos elementos. La adaptación a/en la vejez es percibida como un proceso que puede tomar muchas direcciones. Debido a la complejidad de los factores involucrados, existen muchas respuestas posibles para el envejecimiento. De igual modo, los ancianos mantienen muchos de sus roles y estatus mediante la continuidad del desempeño de sus habilidades para adaptarse mejor a la etapa de la vejez (Covey, 198353).

En un trabajo realizado por Blaum y Jarvik $(1974)^{54}$, interesados por estudiar las diferencias entre ancianos en función del nivel educativo poseido, llegaron a la conclusión

52. Atchley, C. R. (1971). Retirement and leisure participation: Cotninuity or crisis?. The Gerontologist, 11, pp. 13-17.

53. Covey, C. H. (1983). Higher Education and Older People: some Theoretical considerations, Part. I. Educational Gerontology, 9, pp. 1-13.

54. Blaum, J. E. y Jarvik, L. F. (1974). Intellectual performance as a function of octogenarians as a function of education and initiability. Human Development, 17, pp. 364-375. 
de que existían diferencias entre éstos, a favor de los mejor y más «educados», quienes presentaron mejores resultados en las pruebas aplicadas. Sin embargo, la explicación de este hecho para los autores, se debe, más que a la educación en sí misma, a la configuración, por medio de ésta, de estilos de vida continuados que favorecen el mantenimiento de la actividad mental. Interpretación que va en la línea de la Teoría de la Continuidad.

Más aún, para los defensores de la teoría de la continuidad, ésta contempla de forma completa y en toda su variedad la realidad del envejecimiento. De ahí la etiqueta de continuidad puesto que la vejez no es vista como un período separado de la vida (Donahue, 1950: 4355), sino como una continuación de diferentes conjuntos de patrones cognitivos, comportamentales y actitudinales previos, que se han ido forjando a lo largo de toda la vida.

Por otro lado, Fox (1981) ${ }^{56}$ menciona dos tipos de dimensiones que definen el concepto de continuidad y que pueden ayudar a su mejor comprensión. El primero tiene que ver con el concepto sociológico de «carrera» (retomado por los psicólogos del ciclo vital (lifespan) como 'carrera normativa') y el segundo hace referencia al grado de determinismo que la teoría lleva implícito.

En el primer caso, el concepto de carrera, normalmente utilizado para describir dinámicas ocupaciones y/o familiares (matrimonio, nacimiento de hijos, acceso al primer empleo; retiro; viudedad...), implica una progresión ordenada o secuencia de pasos, cada uno de los cuales está relacionado con el paso precedente y con el paso o etapa subsiguiente. Para Fox (1981) las carreras vitales pueden ser ordenadas o desordenadas, dependiendo del grado de conexión entre las fases. Las etapas pueden estar dentro o fuera de la secuencia, las transiciones de una etapa o paso a otro pueden ser, diferencialmente, más próximas o más lejanas en el tiempo, de unos individuos a otros, en función de las presiones ambientales y sociales; la progresión a través de la 'carrera' puede ser vertical u horizontal, etc. (Fox, 1981: 99-101).

La segunda dimensión a la que aludíamos, y que ha sido uno de los flancos donde mayores críticas se han vertido sobre la teoría, tiene que ver con la visión de la continuidad como una forma inflexible, prefijada, de ciertos rasgos, preferencias, conductas..., que, de algún modo, están condicionando el tipo de envejecimiento de las personas. De este modo, podríamos parafrasear el dicho popular: «dime cómo eres ahora y te diré como serás de viejo», si atendemos a la observación del tipo de rasgos de personalidad preprogramados y prácticamente fijados en las etapas previas de la infancia, adolescencia y/o madurez.

Abundando más en la crítica hacia esta teoría, algunos autores consideran que la «discontinuidad» es tan característica de los procesos de envejecimiento como lo es la continuidad y que la continuidad puede ser maladaptativa en muchos casos, debido a los vertiginosos cambios que se están produciendo en las sociedades occidentales.

Desde el punto de vista de la participación educativa de los ancianos, la Teoría de la Continuidad entiende que esta participación debe producirse como una forma de adaptación a la vejez y una expresión más de la variedad de respuestas válidas y posibles. De este modo, se entiende que si los individuos son prácticamente los mismos en la madu-

55. Donahue, W. (1960). Preparation for living in the later years. Adult Education, 1, pp. 43-51.

56. Fox, H. J. (1981). Perspectives on the continuity perspective. International Journal of Aging and Human Development, Vol. 14 (2), 1981-82, pp. 97-115. 
rez que en la vejez, con las mismas necesidades psicológicas y sociales, la participación socioeducativa en esta última tendrá mucho que ver con la participación socioeducativa previa. Esto es, La Teoría de la Continuidad sugiere que aquellos ancianos mejor predispuestos para participar en educación serán aquellos que tienen establecidos roles educativos y patrones de conducta en ese sentido, conformados en épocas pretéritas. Este es un aspecto relevante para la práctica educativa al favorecer y potenciar la necesidad de educación permanente a lo largo de toda la vida y en especial durante la época laboral activa mediante, por ejemplo, programas y diseños de preparación educativa a la jubilación.

El apoyo empírico de esta teoría no es abundante. En un estudio realizado por el mismo Covey (1980) sobre la participación de los ancianos en un programa universitario descubrió que los estudiantes ancianos que tenían antecedentes educativos a lo largo de la vida tanto de forma informal como formal participaron en este programa simplemente como una continuación de lo que ellos habían estado haciendo siempre (Covey, 1980) ${ }^{57}$.

Para Covey (1980) este dato, mientras pueda verse generalizado en otras poblaciones, presta apoyo a la Teoría de la Continuidad. Del mismo modo, mantiene al respecto que, en relación con los antecedentes educativos de la participación:

1. Se puede argumentar que los ancianos más y mejor educados (con mayor nivel instructivo o formativo) tienen mejor establecido el rol de estudiante. Las personas que tienen asumido ese rol conocen sus obligaciones, sus derechos y las expectativas que encierra.

2. Los ancianos llegan a incorporar el rol de estudiante dentro de su repertorio de roles, esto es, la experiencia en el rol de estudiante y el rol mismo son parte de la estructura social de la persona.

3. Puesto que estos antecedentes son a largo plazo la transición dentro del rol de estudiante, pasada la edad de 60 años, se produce simplemente a continuación de actividades educativas desarrolladas, de algún modo, durante toda la vida (Covey, 1980: p. 105).

De otra forma, la transición, adquisición y resultado de los logros y resultados en el rol de estudiante es relativamente fácil, puesto que el vacío entre el ser y el no ser un estudiante es relativamente pequeño para aquellos que participan de ese rol. En cambio, el vacío se ensancha para aquellos ancianos que tienen poca o ninguna experiencia en educación (Covey, 1983) ${ }^{58}$.

Finalmente, esta perspectiva entiende que los ancianos poseen un potencial, incluso intelectual, que está sujeto aún, a desarrollo y crecimiento. De este modo, dice Covey (1983: 104-106) «la Teoría de la Continuidad nos lleva en una dirección satisfactoria para explicar la participación de los ancianos en educación superior».

57. Véase Covey, H. C. (1980). An exploratory study of the adquisition of a college student role by older people. Gerontologist, 20, pp. 173-181; Covey, H. C. (1981). A reconceptualization of Continuity Theory: some preliminary thoughts. Gerontologist, 21, pp. 628-633.

58. Covey, H. C. (1983). Op. cit., pp. 99. 


\section{DISCUSIÓN}

El análisis efectuado más arriba nos ha conducido, finalmente, a la revisión de la Teoría de la Continuidad, donde el peso específico, como veíamos, es depositado en los antecedentes biográficos que han configurado y configuran determinados estilos vitales en los individuos y que permanecen (sujetos, eso sí, a ciertas modificaciones) a lo largo del tiempo. Precisamente son esos estilos de vida los que, pensamos, condicionan gran parte de las decisiones y también de las acciones de participar o no en actividades socioculturales y socioeducativas.

Parece pues claro que lo que se está intentando promover y comprobar científicamente actualmente es la existencia de procesos individuales diferenciadores de envejecimiento. Procesos en los que están conjugadas variables como por ejemplo las historias vitales, la propia experiencia, el ambiente ecológico, etc. y donde la variable edad juega un papel importante pero no universalmente decisivo ni decisorio. En este último caso, los evidentes esfuerzos que hoy día la gerontología (en cualquiera de sus ramificaciones disciplinares) está realizando para relativizar la importancia concedida a la variable edad biológica, frente a la idea hasta ahora comúnmente aceptada de que la edad natural, cronológica, del individuo marcaba gran parte de las pautas negativas asociadas al proceso de envejecimiento, no puede ocultar la necesidad de dedicar similares esfuerzos por relativizar, flexibilizar y reajustar la significación cultural de otro tipo de «edades» como la social y la educativa. Eso es así puesto que, tradicionalmente, la educación ha sido concebida como una actividad terminal asociada a determinadas etapas de edad cronológica a partir de la cual comienzan nuevas etapas o actividades diferentes y es muy difícil la vuelta atrás. Esto es, los sistemas sociales, legales, laborales y educativos han estado, y lo están aún, sujetos a esquemas rígidos en los que la edad del sujeto constituye el punto de partida y, a veces, de llegada. De hecho, en los sistemas educativos, el ingreso y el regreso en la mayoría de los niveles sigue hoy día vinculado a normas de edad determinadas institucionalmente. De este modo, la participación social y educativa de los sujetos está modulada por la asignación de roles y estatus asignados a los individuos, lo que conlleva generalmente a la asignación de oportunidades y recursos sociales y a la diferenciación de las interrelaciones sociales que en ella se produce.

En definitiva, la transición desde los centros educativos orientados fundamentalmente para jóvenes a la integración en los mismos, o en otros específicos, de personas adultas-ancianas, tendrá que superar numerosos obstáculos para vencer la segregación asociada al factor edad. Entre estos obstáculos podemos apuntar: el desarrollo de fórmulas y estrategias para que la asunción social y personal (por parte del propio sujeto anciano) del papel y el estatus de «estudiante» sea efectiva (evidentemente la significación del término 'estudiante' que hemos utilizado aquí, puede ser o no, diferente que para el sujeto estudiante joven); el desarrollo de estrategias, programas y diseños educativos adecuados realmente a las necesidades de este sector poblacional; desarrollo de la investigación gerontopedagógica sobre los procesos de envejecimiento y su incidencia sobre la participación educativa, etc. 